\title{
Biomarkers of endothelial dysfunction in alcoholic hepatitis
}

\author{
Tiffany $\mathrm{Wu}^{1} \cdot$ Vijay Shah ${ }^{1}$
}

Received: 31 March 2021 / Accepted: 29 April 2021 / Published online: 27 May 2021

(c) Asian Pacific Association for the Study of the Liver 2021

Alcoholic hepatitis (AH) is a severe form of alcohol-associated liver disease (ALD) with high short-term mortality attributed to hepatic decompensation, renal failure, bacterial infection, or portal hypertension [1,2]. Although mechanisms of pathogenesis require further refinement, excessive alcohol consumption is believed to increase intestinal permeability, disrupt gut flora, and enable translocation of bacteria that induce liver injury and inflammation [3]. Yet, even in the absence of cirrhosis, individuals with AH may develop sequelae of advanced liver disease including portal hypertension. Endothelial dysfunction plays a predominant role in pathogenesis, with damaged endothelium expressing a provasoconstrictive, prothrombotic, proinflammatory, and proangiogenic phenotype that leads to downstream pathophysiology of portal hypertension. Given multiple mechanisms contributing to disease progression, pathogenic molecules of endothelial cell disruption and dysregulation have potential to serve as non-invasive biomarkers of disease severity and prognostication in $\mathrm{AH}$.

Various mechanisms have been identified in the multifactorial phenotypic alterations of endothelial cells. Specifically, endothelial cell dysfunction has been associated with increased intrahepatic vascular resistance due to altered nitric oxide synthase function resulting in reduced nitric oxide production by sinusoidal endothelial cells $[4,5]$. In addition to this provasoconstrictive effect, sinusoidal thrombosis has been implicated through the formation of neutrophil extracellular traps (NETs) that may initiate or propagate coagulation [6]. Hilscher et al. further clarified this association by identifying the role of volume and pressure impact within sinusoids modulating NET-induced fibrin thrombi, thereby linking this prothrombotic effect to the pathogenesis of portal hypertension [7]. Certainly, sinusoidal endothelial cells mediate proinflammatory signaling in hepatic immune

Vijay Shah

shah.vijay@mayo.edu

1 Division of Gastroenterology and Hepatology, Mayo Clinic, Rochester, MN, USA response [8] and participate in crosstalk with contractile hepatic stellate cells in the early steps of liver fibrogenesis $[9,10]$. Concurrent to fibrosis, endothelial cells also participate in angiogenesis through multiple postulated mechanisms, including autocrine signaling stimulated by structural changes in sinusoids that further promote fibrogenesis and contribute to portal hypertension [10]. Much of the modeling for endothelial damage and consequent changes leading to portal hypertension have been established in the setting of chronic liver disease. However, we are increasingly recognizing similar pathogenesis in severe alcoholic hepatitis.

In this issue of Hepatology International, Blaya et al. [11] take the necessary step to unify the pathogenic mechanisms of $\mathrm{AH}$ and development of portal hypertension by demonstrating correlation between increased markers of endothelial dysfunction and outcomes of AH. They also propose the use of pathogenic markers of endothelial cell function as promising biomarkers of disease.

The authors first presented an analysis of multiple markers of endothelial dysfunction, including vascular cell adhesion protein (VCAM)-1, intercellular adhesion molecule (ICAM)-1, E-selectin, P-selectin, von Willebrand factor (vWF), von Willebrand factor domain A2 (vWF-A2), and ADAMST13. They profiled plasma levels of these biomarkers from a test and validation cohort of patients with histologically proven alcoholic hepatitis compared to patients with compensated alcoholic cirrhosis, heavy drinking but no liver disease, and healthy controls, and then evaluated biomarker association with disease severity and clinical outcomes. Notably, they identified that patients with severe $\mathrm{AH}$ and evidence of elevated portal pressures (mean HPVG $18.24 \mathrm{mmHg}$ and $19.18 \mathrm{mmHg}$ in the test and validation cohorts, respectively) demonstrated higher levels of endothelial cell markers compared to controls. Among the various biomarkers, VCAM-1 emerged as the marker with significant increase in $\mathrm{AH}$ patients with greater severity of disease (MELD $\geq 21$ ). The authors also found significant correlation between increased plasma levels of VCAM-1 and overall clinical disease severity and outcomes. Plasma levels of VCAM-1 positively 
correlated with clinical scoring systems such as Maddrey discriminant function (MDF), model for end-stage liver disease (MELD), and age, serum, bilirubin, international normalized ratio, serum creatinine (ABIC). VCAM-1 also correlated with markers of liver injury and inflammation such as plasma C-reactive protein, lipopolysaccharide, and leukocyte count, further emphasizing the role of endotoxinemia and proinflammatory activation in the altered phenotype of damaged endothelial cells.

By defining the relationship between plasma biomarkers and clinical scores of disease severity, the authors highlighted an ongoing need for identification of novel, non-invasive biomarkers of AH. Current gold standard for diagnosis is based on clinical and laboratory findings, with liver biopsy confirmation in cases of uncertainty [12]. Liver biopsy is invasive, carries risk of significant morbidity, and is not readily accessible in follow-up of response to treatment [13]. Clinical decision-making is facilitated by scoring models that apply readily available biochemical measures, such as MELD, MDF, and ABIC, to assess disease severity, treatment response, and mortality. Among these models, the MELD score is recognized to be a more accurate predictor of mortality up to 90 days [14-16]. Unfortunately, presentation with severe AH carries poor short-term prognosis [17], and we have the additional challenge to diagnose earlier or milder forms of $\mathrm{AH}$, risk stratify patients, and prognosticate disease to determine optimal strategies in management. So far, several plasma biomarkers have been identified for characterization of disease severity and prognosis, such as small non-coding or microRNA, long non-coding RNA, cytokines (IL-1B, TNF, and IL-8), and cytokeratins (cytokeratin-18 and cytokeratin-19) [18]. Recently, circulating extracellular vesicle (EV) concentration and sphingolipid cargo signature, which have been implicated in inflammation and overall $\mathrm{AH}$ pathogenesis, have also demonstrated diagnostic and prognostic utility in AH [19]. Incorporation of EV concentration and sphingolipid species has further augmented the performance of the MELD score in predicting 90-day mortality [19]. However, such biomarkers have yet to be translated into clinical practice.

In this study, multivariate Cox regression analysis showed that plasma level of VCAM-1 was an independent predictor of 90-day mortality, when adjusted by ABIC $(p=0.002)$ and ABIC class $(p=0.001)$. When using a cutoff of $5630 \mathrm{ng} /$ $\mathrm{ml}$, higher levels of plasma VCAM-1 predicted short-term mortality in severe AH (AUC 0.71). As a result, the authors further proposed the utility of pathogenic molecules in endothelial dysfunction, specifically VCAM-1, as a clinically useful biomarker that may meet the diagnostic and prognostic needs of managing patients with $\mathrm{AH}$. This correlation with short-term mortality and transplant-free survival may further aid in risk stratification and prioritization of liver transplantation for this population.
This study confirmed previous findings that levels of soluble forms of pathogenic endothelial cell markers are significantly upregulated and correlate with disease severity, clinical parameters, and other inflammatory factors in ALD. Upregulation of VCAM-1 has been known to be a critical indicator of endothelial cell dysfunction in a spectrum of liver disease associated with excess alcohol ingestion, including AH and alcohol-induced cirrhosis [20]. Recently, serum VCAM-1 levels have also been found to be elevated among heavy drinkers without overt liver disease [21], raising its utility as a more sensitive marker of early endothelial cell dysfunction in pathophysiologic inflammation. Among patients with $\mathrm{AH}$, alcohol abstinence for up to 12 months also did not fully reverse endothelial cell changes and elevated levels of plasma markers, suggesting a lasting effect of inflammation [21]. The role of VCAM-1 as an inflammatory mediator of liver disease is also highlighted by its significant elevation in other sequelae of hepatic decompensation, including acute-on-chronic liver failure and hepatorenal syndrome [22, 23].

However, the authors of the study took one step further. Blaya et al. [11] advanced our understanding of endothelial dysfunction by demonstrating the unifying role of elevated biomarkers in pathogenesis of $\mathrm{AH}$ and development of portal hypertension. This study was limited by small sample sizes in both test $(n=67)$ and validation $(n=50)$ cohorts; however, strengthened by the availability of liver biopsies for histological confirmation in all cases of AH. Using approaches to tag or define a signature for the genetic expression of these signaling molecules may further elucidate the pathogenesis of ALD progression. Until then, these recent findings support the argument for measuring circulating levels of pathogenic endothelial cell markers as biomarkers of alcoholic hepatitis, given the unique role of endothelial dysfunction in pathogenesis of alcoholic hepatitis and downstream pathophysiology of portal hypertension.

\section{References}

1. Lucey MR, Mathurin P, Morgan TR. Alcoholic hepatitis. N Engl J Med. 2009;360(26):2758-69. https://doi.org/10.1056/NEJMr a0805786.

2. Singal AK, Kamath PS, Gores GJ, Shah VH. Alcoholic hepatitis: current challenges and future directions. Clin Gastroenterol Hepatol. 2014;12(4):555-64. https://doi.org/10.1016/j.cgh.2013.06.013 ((Quiz e31-2)).

3. Arab JP, Martin-Mateos RM, Shah VH. Gut-liver axis, cirrhosis and portal hypertension: the chicken and the egg. Hepatol Int. 2018;12(Suppl 1):24-33. https://doi.org/10.1007/ s12072-017-9798-x.

4. Rockey DC, Chung JJ. Reduced nitric oxide production by endothelial cells in cirrhotic rat liver: endothelial dysfunction in portal hypertension. Gastroenterology. 1998;114(2):344-51. https://doi.org/10.1016/s0016-5085(98)70487-1. 
5. Shah V, Toruner M, Haddad F, Cadelina G, Papapetropoulos A, Choo K, Sessa WC, Groszmann RJ. Impaired endothelial nitric oxide synthase activity associated with enhanced caveolin binding in experimental cirrhosis in the rat. Gastroenterology. 1999;117(5):1222-8. https://doi.org/10.1016/s0016-5085(99) 70408-7.

6. Massberg S, Grahl L, von Bruehl ML, Manukyan D, Pfeiler S, Goosmann C, Brinkmann V, Lorenz M, Bidzhekov K, Khandagale AB, Konrad I, Kennerknecht E, Reges K, Holdenrieder S, Braun S, Reinhardt C, Spannagl M, Preissner KT, Engelmann B. Reciprocal coupling of coagulation and innate immunity via neutrophil serine proteases. Nat Med. 2010;16(8):887-96. https://doi.org/10. 1038/nm.2184.

7. Hilscher MB, Sehrawat T, Arab JP, Zeng Z, Gao J, Liu M, Kostallari E, Gao Y, Simonetto DA, Yaqoob U, Cao S, Revzin A, Beyder A, Wang RA, Kamath PS, Kubes P, Shah VH. Mechanical stretch increases expression of CXCL1 in liver sinusoidal endothelial cells to recruit neutrophils, generate sinusoidal microthombi, and promote portal hypertension. Gastroenterology. 2019;157(1):193209.e9. https://doi.org/10.1053/j.gastro.2019.03.013.

8. DeLeve LD, Maretti-Mira AC. Liver sinusoidal endothelial cell: an update. Semin Liver Dis. 2017;37(4):377-87. https://doi.org/ 10.1055/s-0037-1617455.

9. Greuter T, Shah VH. Hepatic sinusoids in liver injury, inflammation, and fibrosis: new pathophysiological insights. J Gastroenterol. 2016;51(6):511-9. https://doi.org/10.1007/ s00535-016-1190-4.

10. Thabut D, Shah V. Intrahepatic angiogenesis and sinusoidal remodeling in chronic liver disease: new targets for the treatment of portal hypertension? J Hepatol. 2010;53(5):976-80. https://doi. org/10.1016/j.jhep.2010.07.004.

11. Blaya D, Rubio-Tomás T, Rodrigo-Torres D, Lozano J, Coll M, Argemi J, Altamirano J, Affò S, Morales-Ibanez O, GratacósGinès J, Pose E, Tanguy M, Issoufaly T, Rautou P, Bataller R,Caballería J, Sancho-Bru P. Endothelial dysfunction markers predict short-term mortality in patients with severe alcoholic hepatitis. Hepatology International. 2021.

12. Crabb DW, Bataller R, Chalasani NP, Kamath PS, Lucey M, Mathurin P, McClain C, McCullough A, Mitchell MC, Morgan TR, Nagy L, Radaeva S, Sanyal A, Shah V, Szabo G, NIAAA Alcoholic Hepatitis Consortia. Standard definitions and common data elements for clinical trials in patients with alcoholic hepatitis: recommendation from the NIAAA alcoholic hepatitis consortia. Gastroenterology. 2016;150(4):785-90. https://doi.org/10.1053/j. gastro.2016.02.042.

13. Filingeri V, Francioso S, Sforza D, Santopaolo F, Oddi FM, Tisone G. A retrospective analysis of 1.011 percutaneous liver biopsies performed in patients with liver transplantation or liver disease: ultrasonography can reduce complications? Eur Rev Med Pharmacol Sci. 2016;20(17):3609-17.

14. Dunn W, Jamil LH, Brown LS, Wiesner RH, Kim WR, Menon KV, Malinchoc M, Kamath PS, Shah V. MELD accurately predicts mortality in patients with alcoholic hepatitis. Hepatology. 2005;41(2):353-8. https://doi.org/10.1002/hep.20503.

15. Kamath PS, Kim WR, Advanced Liver Disease Study Group. The model for end-stage liver disease (MELD). Hepatology. 2007;45(3):797-805. https://doi.org/10.1002/hep.21563.

16. Louvet A, Labreuche J, Artru F, Boursier J, Kim DJ, O'Grady J, Trépo E, Nahon P, Ganne-Carrié N, Naveau S, Diaz E, Gustot T, Lassailly G, Cannesson-Leroy A, Canva-Delcambre V, Dharancy S, Park SH, Moreno C, Morgan TR, Duhamel A, Mathurin P. Combining data from liver disease scoring systems better predicts outcomes of patients with alcoholic hepatitis. Gastroenterology. 2015;149(2):398-406.e8. https://doi.org/10.1053/j.gastro.2015.04. 044 ((Quiz e16-7)).

17. Morgan MY. The prognosis and outcome of alcoholic liver disease. Alcohol Alcohol Suppl. 1994;2:335-43.

18. Moreno C, Mueller S, Szabo G. Non-invasive diagnosis and biomarkers in alcohol-related liver disease. J Hepatol. 2019;70(2):273-83. https://doi.org/10.1016/j.jhep.2018.11.025.

19. Sehrawat TS, Arab JP, Liu M, Amrollahi P, Wan M, Fan J, Nakao Y, Pose E, Navarro-Corcuera A, Dasgupta D, Liao CY, He L, Mauer AS, Avitabile E, Ventura-Cots M, Bataller RA, Sanyal AJ, Chalasani NP, Heimbach JK, Watt KD, Gores GJ, Gines P, Kamath PS, Simonetto DA, Hu TY, Shah VH, Malhi H. Circulating extracellular vesicles carrying sphingolipid cargo for the diagnosis and dynamic risk profiling of alcoholic hepatitis. Hepatology. 2021;73(2):571-85. https://doi.org/10.1002/hep.31256.

20. Adams DH, Burra P, Hubscher SG, Elias E, Newman W. Endothelial activation and circulating vascular adhesion molecules in alcoholic liver disease. Hepatology. 1994;19(3):588-94. https://doi. org/10.1002/hep.1840190308.

21. Xia Y, Yang J, Sanyal AJ, Shah VH, Chalasani NP, Yu Q, Zheng $\mathrm{X}, \mathrm{Li}$ W. Persistent hyperactivation of endothelial cells in patients with alcoholic hepatitis. Alcohol Clin Exp Res. 2020;44(5):107587. https://doi.org/10.1111/acer.14331 (Epub 2020 Apr 21).

22. Solé C, Solà E, Morales-Ruiz M, Fernàndez G, Huelin P, Graupera I, Moreira R, de Prada G, Ariza X, Pose E, Fabrellas N, Kalko SG, Jiménez W, Ginès P. Characterization of inflammatory response in acute-on-chronic liver failure and relationship with prognosis. Sci Rep. 2016;31(6):32341. https://doi.org/10.1038/srep32341.

23. Solé C, Solà E, Huelin P, Carol M, Moreira R, Cereijo U, Mas JM, Graupera I, Pose E, Napoleone L, dePrada G, Juanola A, Fabrellas N, Torres F, Morales-Ruiz M, Farrés J, Jiménez W, Ginès P. Characterization of inflammatory response in hepatorenal syndrome: relationship with kidney outcome and survival. Liver Int. 2019;39(7):1246-55. https://doi.org/10.1111/liv.14037.

Publisher's Note Springer Nature remains neutral with regard to jurisdictional claims in published maps and institutional affiliations. 